Author affiliations and support information (if applicable) appear at the end of this article.

Published at jco.org on September 20, 2017

Clinical trial information: NCT01969838.

Corresponding author: Ruben A. Mesa, MD, Mayo Clinic Cancer Center, 5777 E Mayo Blvd, Phoenix, AZ; e-mail: mesa.ruben@ mayo.edu.

(c) 2017 by American Society of Clinical Oncology

$0732-183 \times / 17 / 3534 w-3844 w / \$ 20.00$

\title{
SIMPLIFY-1: A Phase III Randomized Trial of Momelotinib Versus Ruxolitinib in Janus Kinase Inhibitor-Naïve Patients With Myelofibrosis
}

Ruben A. Mesa, Jean-Jacques Kiladjian, John V. Catalano, Timothy Devos, Miklos Egyed, Andrzei Hellmann, Donal McLornan, Kazuya Shimoda, Elliott F. Winton, Wei Deng, Ronald L. Dubowy, Julia D. Maltzman, Francisco Cervantes, and Jason Gotlib

\section{$\begin{array}{llllllll}\text { A } & \text { B } & \text { S } & \text { T } & \text { R } & \text { A } & \text { C } & \text { T }\end{array}$}

\section{Purpose}

We evaluated the efficacy and safety of momelotinib, a potent and selective Janus kinase 1 and 2 inhibitor (JAKi), compared with ruxolitinib, in JAKi-naïve patients with myelofibrosis.

\section{Patients and Methods}

Patients ( $N=432$ ) with high risk or intermediate-2 risk or symptomatic intermediate-1 risk myelofibrosis were randomly assigned to receive 24 weeks of treatment with momelotinib $200 \mathrm{mg}$ once daily or ruxolitinib 20 mg twice a day (or per label), after which all patients could receive open-label momelotinib. The primary end point was a $\geq 35 \%$ reduction in spleen volume at 24 weeks of therapy. Secondary end points were rates of symptom response and effects on RBC transfusion requirements.

\section{Results}

$A \geq 35 \%$ reduction in spleen volume at week 24 was achieved by a similar proportion of patients in both treatment arms: $26.5 \%$ of the momelotinib group and $29 \%$ of the ruxolitinib group (noninferior; $P=.011) . A \geq 50 \%$ reduction in the total symptom score was observed in $28.4 \%$ and $42.2 \%$ of patients who received momelotinib and ruxolitinib, respectively, indicating that noninferiority was not met $(P=.98)$. Transfusion rate, transfusion independence, and transfusion dependence were improved with momelotinib (all with nominal $P \leq$.019). The most common grade $\geq 3$ hematologic abnormalities in either group were thrombocytopenia and anemia. Grade $\geq 3$ infections occurred in $7 \%$ of patients who received momelotinib and $3 \%$ of patients who received ruxolitinib. Treatmentemergent peripheral neuropathy occurred in $10 \%$ of patients who received momelotinib (all grade $\leq 2$ ) and $5 \%$ of patients who received ruxolitinib (all grade $\leq 3$ ).

\section{Conclusion}

In JAKi-naïve patients with myelofibrosis, 24 weeks of momelotinib treatment was noninferior to ruxolitinib for spleen response but not for symptom response. Momelotinib treatment was associated with a reduced transfusion requirement.

\section{J Clin Oncol 35:3844-3850. (C) 2017 by American Society of Clinical Oncology}

\section{INTRODUCTION}

Myelofibrosis (MF) is a myeloproliferative neoplasm characterized by anemia, extramedullary hematopoiesis, splenomegaly, and often-debilitating constitutional symptoms. ${ }^{1}$ Approximately $90 \%$ of patients with MF harbor mutations in JAK2, CALR, or MPL leading to constitutive activation of Janus kinase (JAK)-signal transducers and activators of transcription signaling. ${ }^{2-4}$ Currently, the only US Food and Drug Administration-approved treatment of MF is ruxolitinib, a JAK inhibitor (JAKi) that has demonstrated therapeutic benefit in the control of symptomatic splenomegaly and constitutional symptoms in patients with or without the JAK2 mutation. ${ }^{5-7}$ Common hematologic adverse events (AEs) with ruxolitinib treatment include anemia and thrombocytopenia, which can lead to dose reductions or treatment interruptions. ${ }^{6,7}$ Therefore, an unmet need exists for new therapeutic options that improve responses while reducing anemia and other toxicities associated with currently available therapies.

Momelotinib is an investigational, oral, small-molecule inhibitor of JAK1/2 with selectivity over other tyrosine and serine/threonine kinases. ${ }^{8-10}$ In preclinical studies, momelotinib has also demonstrated 
inhibition of the bone morphogenic protein receptor kinase activin A receptor, type I (ACVR1)-mediated expression of hepcidin in the liver, thereby increasing iron availability for erythropoiesis. ${ }^{10}$ Thus, there is a rationale for the use of momelotinib in patients with MF where anemia remains a challenging clinical problem. A phase I/II study evaluating momelotinib in MF demonstrated reduction in spleen volume, improvement of MF-associated symptoms, and reduction of $\mathrm{RBC}$ transfusion requirements in patients with MF. ${ }^{11}$ In this analysis, we report the results of the phase III trial, Momelotinib Versus Ruxolitinib in Subjects With Myelofibrosis (SIMPLIFY-1) in JAKi-naïve patients with MF that compares the efficacy and safety of momelotinib versus ruxolitinib. A companion trial, SIMPLIFY-2, compares momelotinib with best available therapy in MF in patients who experienced either suboptimal responses or toxicity to ruxolitinib. ${ }^{12}$

\section{PATIENTS AND METHODS}

\section{Patient Eligibility, Stratification, and Treatment}

Patients were $\geq 18$ years of age with palpable splenomegaly $\geq 5 \mathrm{~cm}$ below the left costal margin and a confirmed diagnosis of primary MF (WHO criteria ${ }^{13}$ ) or post-polycythemia vera or post-essential thrombocythemia MF (International Working Group for Myelofibrosis Research and Treatment [IWG-MRT] criteria ${ }^{14}$ ). Patients were classified as International Prognostic Scoring System ${ }^{15}$ high risk, intermediate-2 risk, or intermediate-1 risk with symptomatic splenomegaly or hepatomegaly or anemia (hemoglobin $[\mathrm{Hb}]<10.0 \mathrm{~g} / \mathrm{dL}$ ) and/or unresponsive to available non-JAKi therapy. Within 14 days before the first dose of study treatment, the following laboratory tests were required: absolute neutrophil count $\geq 0.75 \times 10^{9} / \mathrm{L}$ in the absence of growth factor therapy in the prior 7 days; platelet count $\geq 50 \times 10^{9} / \mathrm{L}\left(\geq 100 \times 10^{9} / \mathrm{L}\right.$ if AST or ALT $\geq 2 \times$ upper limit of normal [ULN]) in the absence of platelet transfusion(s) or thrombopoietin mimetics in the prior 7 days; peripheral blood blasts $<10 \%$, AST and ALT $\leq 3 \times \mathrm{ULN}$ ( $\leq 5 \times \mathrm{ULN}$ if liver is involved by extramedullary hematopoiesis as judged by the investigator or if related to iron chelator therapy that was started within the prior 60 days); and creatinine clearance $\geq 45 \mathrm{~mL} / \mathrm{min}$ and direct bilirubin $\leq 2.0 \times$ ULN Patients had Eastern Cooperative Oncology Group performance status ${ }^{16} \leq 2$ and life expectancy $>24$ weeks. Patients with prior use of a JAKi, prior splenectomy, spleen irradiation $<3$ months before the first dose of study treatment, certain cancers (history or concurrent disease), or uncontrolled intercurrent illness that would limit study compliance as judged by the treating physician, or who were eligible for allogenic stem-cell transplantation, were excluded.

This study had a 24-week double-blind double-dummy treatment phase. Patients were randomly assigned by an interactive web-based response system 1:1 to either momelotinib (200 mg once daily) or ruxolitinib $20 \mathrm{mg}$ twice a day (or modified as per label). Treatment assignment was stratified by transfusion dependence (yes or no; defined as $\geq 4$ units of RBCs or $\mathrm{Hb}$ level $<8 \mathrm{~g} / \mathrm{dL}$ in the 8 weeks before random assignment, excluding patients associated with clinically overt bleeding) and by platelet count $\left(<100 \times 10^{9} / \mathrm{L}, \geq 100 \times 10^{9} / \mathrm{L}\right.$ and $\leq 200 \times 10^{9} / \mathrm{L}$, or $\left.>200 \times 10^{9} / \mathrm{L}\right)$. After completion of the double-blind treatment phase, patients were eligible to receive momelotinib in an open-label phase.

\section{Study Design}

This was a multicenter, randomized, double-blind phase III clinical trial. The study was approved by institutional review boards/independent ethics committees, and all participants provided written consent. Clinic visits were at screening (initial visit and ensuing assessments to determine eligibility), baseline (visit $<10$ days before random assignment), random assignment (visit for administration of first dose of study drug), and every
2 weeks during the double-blind phase. Patients completed the modified Myeloproliferative Neoplasm Symptom Assessment Form Total Symptom Score (TSS) using an electronic diary daily from screening through the double-blind phase. Eastern Cooperative Oncology Group performance status and laboratory assessments were done during clinic visits, and abdominal magnetic resonance imaging (MRI) or computed tomography (CT) scans were performed every 12 weeks. A record of all transfusions received during screening and throughout the study was kept in the patients' diaries.

\section{Statistical Analysis and End Points}

The primary end point was a reduction of $\geq 35 \%$ in spleen volume from baseline at week 24 (spleen response rate, SRR24), as assessed by MRI or CT scan and evaluated by a blinded central reader. The primary hypothesis was that momelotinib is noninferior to ruxolitinib. On the basis of the assumption of the common treatment effect on SRR being 34\% (lower bound of the $95 \% \mathrm{CI}$ on the ruxolitinib effect on SRR) that was observed in the Controlled Myelofibrosis Study With Oral JAK Inhibitor Treatment $(\text { COMFORT }-1)^{7}$, a total sample size of 420 provides $>90 \%$ power for testing the noninferiority hypothesis on SRR24. Noninferiority of momelotinib was determined by whether the lower bound of the two-sided $95 \% \mathrm{CI}$ for the noninferiority difference (SRR24 of momelotinib $-0.6 \times$ SRR24 of ruxolitinib) was $>0$ and was calculated based on the stratum-adjusted Cochran-Mantel-Haenszel (CMH) proportion.

Four end points at week 24 were designated as secondary end points for which sequential testing was performed in the order listed to control the type 1 error rate: TSS response rate (proportion of patients who achieved a $\geq 50 \%$ reduction from baseline to week 24 on the basis of the modified Myeloproliferative Neoplasm Symptom Assessment Form TSS diary); RBC transfusion-independence rate (proportion of patients who were transfusion-independent at week 24 [absence of RBC transfusions and no $\mathrm{Hb}<8 \mathrm{~g} / \mathrm{dL}$ in the prior 12 weeks]); RBC transfusion-dependence rate (proportion of patients who were transfusion-dependent $[\geq 4$ units of RBC transfusions, or $\mathrm{Hb}<8 \mathrm{~g} / \mathrm{dL}$ in the prior 8 weeks]); and rate of RBC transfusion (average number of RBC units per subject-month during treatment). Most secondary end points were evaluated similarly to the primary end point (CMH approach), with the exception of the RBC transfusion rate, which was analyzed using a negative binomial regression method adjusted for stratification factors with an offset parameter to account for follow-up time. The primary end point analysis served as the gatekeeper for the secondary end point analyses, such that only if the primary efficacy hypothesis was rejected could the formal, statistical testing be undertaken for the four secondary efficacy end points sequentially in the order listed above. If a null hypothesis was not rejected, formal sequential testing was stopped and only nominal significance would be cited for the remaining secondary end points.

Exploratory end points included overall response rate, which was defined as the proportion of patients who achieve a complete response (CR) or partial response (PR) according to the IWG-MRT and/or European LeukemiaNet $(\mathrm{ELN})^{17}$ criteria. The composite clinical improvement rate was defined as the proportion of patients who achieved CR and PR on the basis of IWG-MRT and/or ELN response criteria or who achieved anemia response, MRI/CT spleen response, or TSS response at week 24.

Efficacy end points were analyzed in the intent-to-treat population consisting of all patients randomly assigned, except TSS response rate, which was analyzed in all randomly assigned patients with baseline TSS $>0$ or with baseline TSS of 0 , but week 24 TSS missing or $>0$. Patients without baseline and/or week 24 visit assessments for the corresponding end point were regarded as splenic nonresponders, TSS nonresponders, not transfusion independent, or transfusion dependent at week 24. Differences between treatment arms for continuous end points were assessed using analysis of covariance, with treatment and stratification factors as factors and baseline values as covariates. Differences between treatment arms were compared using $\mathrm{CMH}$ approach after adjusting for stratification factors. Sensitivity analyses for the primary end point included analysis on per-protocol analysis set, using last observation carried forward for missing data, unstratified method, and fixed margin method. Treatment-emergent AEs were monitored 
continuously, graded for severity and relationship to treatment, and summarized by treatment group.

\section{RESULTS}

\section{Patient Characteristics and Disposition}

Between December 6, 2013 and September 12, 2016, 127 study centers enrolled patients in this international, multicenter trial. A total of 432 patients underwent random assignment, of whom 215 were assigned to receive momelotinib and 217 were assigned to receive ruxolitinib; 214 in the momelotinib group and 216 in the ruxolitinib group received one or more doses of study drug. Treatment discontinuation was reported for $18.6 \%$ and $7.4 \%$ of patients in the momelotinib and ruxolitinib groups, respectively. The baseline and demographic characteristics were similar between groups (Table 1). Most patients were white $(82.6 \%)$, male $(56.5 \%)$, and $\geq 65$ years of age $(57.2 \%)$, and $56.5 \%$ had primary MF. The median (first-third quartiles, Q1-Q3) time since diagnosis was 1.5 years (0.4 to 3.9 years). The 24-week double-blind phase was completed by 376 patients (momelotinib $(\mathrm{n}=175)$, ruxolitinib $(\mathrm{n}=201) ; 368$ patients continued in the open-label phase of the study (171 from the momelotinib group and 197 from the ruxolitinib group switched to momelotinib). Patient disposition and availability for assessments are shown in Appendix Figure Al (online only).

\section{Spleen Assessments}

Overall, 184 patients who received momelotinib and 204 patients who received ruxolitinib had assessments available for SRR24. Figure 1 shows individual patients' spleen response as measured by MRI or CT scan. Spleen volume was reduced $\geq 35 \%$ from baseline in $26.5 \%$ (57 of 215) of patients who received momelotinib and $29.0 \%$ (63 of 217 ) of patients who received ruxolitinib, with a noninferiority proportion difference of 0.09 ( $95 \%$ CI, 0.02 to 0.16). Because the lower bound of the two-sided 95\% CI was $>0$, momelotinib met the primary end point of noninferiority to ruxolitinib $(P=.011)$. Sensitivity analyses were consistent with the primary analysis.

\section{Secondary End Points}

A total of 175 patients who received momelotinib and 190 patients who received ruxolitinib had assessments available for TSS. Fewer patients who received momelotinib $(28.4 \%, 60$ patients) had a reduction in TSS of $\geq 50 \%$ from baseline compared with those who received ruxolitinib $(42.2 \%, 89$ patients), indicating less symptomatic improvement in patients who received momelotinib (Fig 2A). The noninferiority proportion difference was 0.00 (95\% CI, -0.08 to 0.08$)$; because the lower bound of the two-sided $95 \%$ CI was not $>0$, noninferiority of momelotinib to ruxolitinib was not met $(P=.98)$. Median scores for individual symptoms were generally low at baseline (medians ranged from 2 to 4 of a possible 10). Greatest improvements were seen in pain under left ribs, night sweats, and itching, and larger relative changes were seen with ruxolitinib compared with momelotinib (Fig 2B).

Because noninferiority of momelotinib to ruxolitinib on response rate in TSS at week 24 was not met, formal sequential

\begin{tabular}{|c|c|c|}
\hline Characteristic & $\begin{array}{l}\text { Momelotinib } \\
(\mathrm{n}=215)\end{array}$ & $\begin{array}{l}\text { Ruxolitinib } \\
(n=217)\end{array}$ \\
\hline Mean age, years (SD) & $65.0(10.67)$ & $64.4(10.59)$ \\
\hline Male & $124(57.7)$ & $120(55.3)$ \\
\hline Mean body mass index, $\mathrm{kg} / \mathrm{m}^{2}$ (SD) & $24.9(4.02)$ & 25.3 (3.99) \\
\hline \multicolumn{3}{|l|}{ Race } \\
\hline White & 179 (83.3) & $178(82.0)$ \\
\hline Black & $2(0.9)$ & $2(0.9)$ \\
\hline Asian & $17(7.9)$ & $20(9.2)$ \\
\hline Ethnicity Hispanic or Latino & $6(2.8)$ & $4(1.8)$ \\
\hline \multicolumn{3}{|l|}{ Myelofibrosis subtype } \\
\hline Primary & $128(59.5)$ & $116(53.5)$ \\
\hline Post-polycythemia vera & $48(22.3)$ & $50(23.0)$ \\
\hline Post-essential thrombocythemia & $39(18.1)$ & $51(23.5)$ \\
\hline \multicolumn{3}{|l|}{ IPSS risk category } \\
\hline Intermediate-1 & 46 (21.4) & $43(19.8)$ \\
\hline Intermediate-2 & 76 (35.3) & 67 (30.9) \\
\hline High & $93(43.3)$ & 107 (49.3) \\
\hline \multicolumn{3}{|l|}{ JAK2V617F mutation } \\
\hline Previously tested & $187(87.0)$ & $194(89.4)$ \\
\hline Positive & $125(58.1)$ & $141(65.0)$ \\
\hline Negative & $61(28.4)$ & $53(24.4)$ \\
\hline Not previously tested & $28(13.0)$ & $23(10.6)$ \\
\hline TSS score, mean (SD) & $19.4(13.18)$ & $17.9(11.47)$ \\
\hline \multicolumn{3}{|l|}{ ECOG performance status } \\
\hline 0 & 76 (35.3) & $72(33.2)$ \\
\hline 1 & $122(56.7)$ & $120(55.3)$ \\
\hline 2 & $17(7.9)$ & 25 (11.5) \\
\hline Mean $\mathrm{Hb}, \mathrm{g} / \mathrm{dL}(\mathrm{SD})$ & $10.6(2.10)$ & $10.7(2.38)$ \\
\hline $\mathrm{Hb} \geq 8 \mathrm{~g} / \mathrm{dL}$ & $186(86.5)$ & $195(89.9)$ \\
\hline Transfusion independent & $147(68.4)$ & $152(70.0)$ \\
\hline Transfusion dependent & $53(24.7)$ & $52(24.0)$ \\
\hline Mean platelet count, $\times 10^{3} / \mu \mathrm{L}(\mathrm{SD})$ & $301.1(207.03)$ & $301.5(255.88)$ \\
\hline $\begin{array}{l}\text { Mean absolute neutrophil count, } \\
\quad \times 10^{3} / \mu \mathrm{L} \text { (SD) }\end{array}$ & 12.0 (13.39) & $11.3(11.04)$ \\
\hline \multicolumn{3}{|c|}{$\begin{array}{l}\text { NOTE. Data presented as No. (\%) unless otherwise noted. No significant } \\
\text { between-group differences in any of the listed baseline characteristics. } \\
\text { Abbreviations: ECOG, Eastern Cooperative Oncology Group; Hb, hemoglobin; } \\
\text { IPSS International Prognostic Scoring System: TSS total symptom score. }\end{array}$} \\
\hline
\end{tabular}

testing was stopped and only nominal significance was reported for the remaining $\alpha$-controlled secondary end points. More patients who received momelotinib were transfusion independent at week 24 (66.5\%) compared with the ruxolitinib group (49.3\%; nominal $P<.001$; Fig 3A). Fewer patients who received momelotinib were transfusion dependent at week $24(30.2 \%)$ compared with those who received ruxolitinib (40.1\%; nominal $P=.019$; Fig $3 \mathrm{~B})$. The median rate of RBC transfusion through week 24 was 0 units/mo in the momelotinib group compared with 0.4 units/mo in the ruxolitinib group (nominal $P<.001$; Fig 3C).

More patients achieved two or more of the predefined end points (SRR24, TSS response, or transfusion independence) at week 24 in the momelotinib group (38.6\%) than in the ruxolitinib group (34.6\%). The proportion of patients who achieved all three end points was also higher with momelotinib (10.2\%) than with ruxolitinib (7.8\%; Fig 4).

\section{Exploratory End Points}

The best overall response rate at any time on the basis of IWGMRT and/or ELN criteria was 5.1\% (11 patients, two with CR and nine with PR) in the momelotinib group and 3.2\% (seven patients, two with $\mathrm{CR}$ and five with PR) in the ruxolitinib group. Composite clinical improvement was numerically greater in the momelotinib 


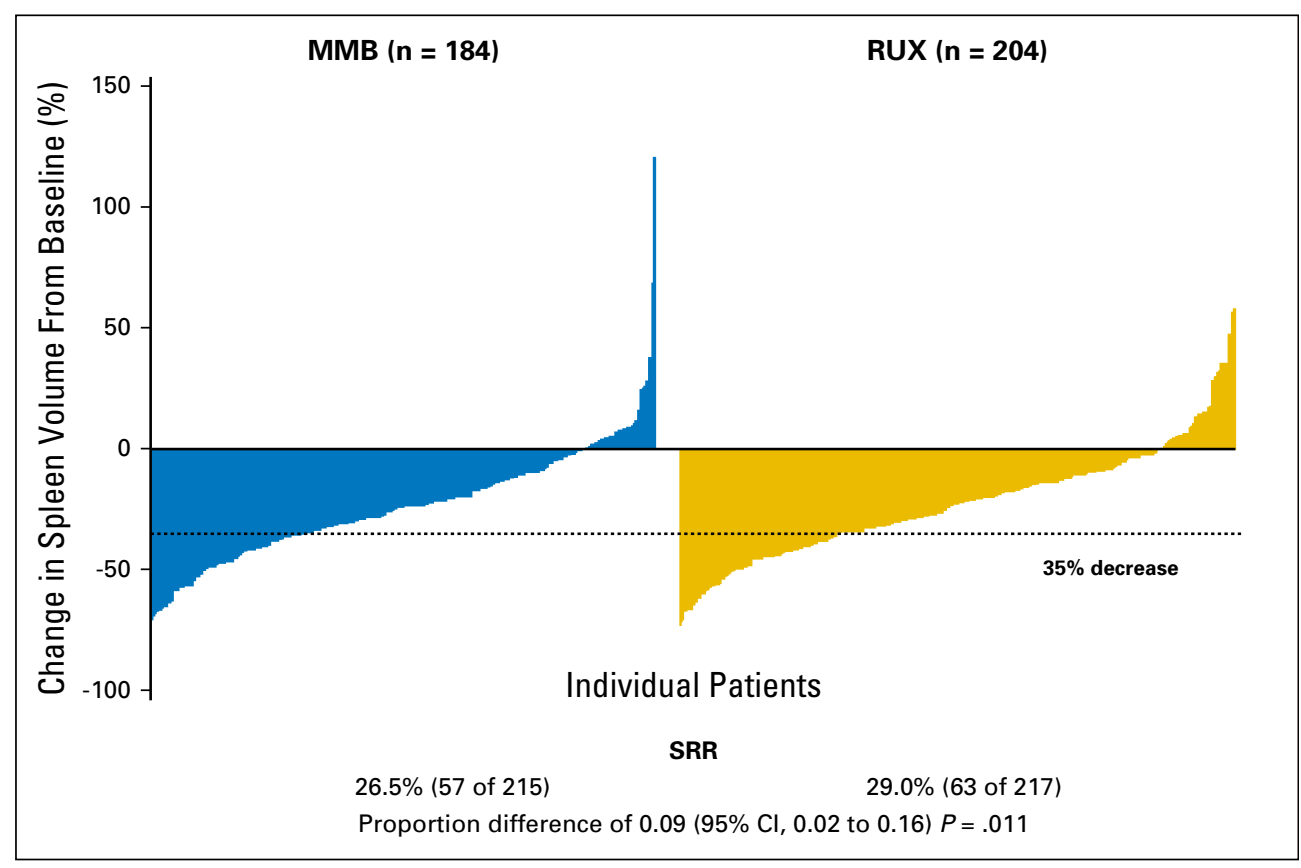

Fig 1. Change in spleen volume and spleen response rate (percentage of patients with $a \geq 35 \%$ reduction in spleen volume) at week 24. $M M B$, momelotinib; RUX, ruxolitinib; SRR, spleen response rate.

group at $35.3 \%$ (76 patients) compared with the ruxolitinib group at $27.2 \%$ (59 patients).

\section{Safety}

During the 24-week treatment phase, the mean duration of exposure to study drug was 21.3 weeks (range, 0.3 to 26.1 weeks) for momelotinib and 23.3 weeks (range, 1.3 to 26.9 weeks) for ruxolitinib; $26.2 \%$ of patients who received momelotinib and $56.0 \%$ of patients who received ruxolitinib had dose reductions or interruptions of active study drug, most frequently for AEs $(17.3 \%$ and 35.6\%, respectively). Most patients (momelotinib, 92.1\%; ruxolitinib, 95.4\%) had one or more AE. The most common treatment-emergent AEs are listed in Table 2. AEs that were grade $\geq 3$ were reported for $35.5 \%$ of patients who received momelotinib and $43.5 \%$ of patients who received ruxolitinib; the most commonly reported grade 3 or 4 AEs with momelotinib were thrombocytopenia $(7.0 \%)$, anemia $(5.6 \% \%)$, and diarrhea, hypertension, and neutropenia $(2.8 \%$ each). The most commonly reported grade 3 or 4 AEs with ruxolitinib were anemia $(23.1 \%)$, neutropenia and thrombocytopenia (4.6\% each), and hypertension (4.2\%). Serious AEs were reported for $22.9 \%$ of patients who received momelotinib and $18.1 \%$ of patients who received ruxolitinib. AEs that lead to discontinuation of active study drug were reported for $13.1 \%$ of patients who received momelotinib and $5.6 \%$ of patients who received ruxolitinib. AEs that lead to dose reduction or temporary interruption of active study drug were reported for $17.8 \%$ of patients who received momelotinib and $36.6 \%$ of patients who received ruxolitinib.

A smaller proportion of patients died or had leukemic transformation in the momelotinib group (15 patients $[7.0 \%])$ compared with the ruxolitinib group (20 patients [9.2\%]), although the difference was not statistically significant. Deaths were reported for seven $(3.3 \%)$ patients who received momelotinib (enteritis, mesenteric vein thrombosis, death, sudden death, sepsis, renal failure, and aortic dissection [one patient each]) and seven (3.2\%) patients who received ruxolitinib (melena, sepsis, pneumonia, head injury, acute myeloid leukemia, recurrent mantle cell lymphoma, and coma [one patient each]). Transformation to acute myeloid leukemia occurred in one patient who received momelotinib (grade 4) and two patients who received ruxolitinib (grade 3 and grade 5).

Peripheral neuropathy was reported in both groups (momelotinib, 22 patients [10.3\%], 28 events; ruxolitinib, 10 patients [4.6\%], 11 events). Two patients in the momelotinib group and one patient in the ruxolitinib group had peripheral neuropathy at baseline. The most frequently reported term was peripheral sensory neuropathy $(8.4 \%$ in the momelotinib group and $4.6 \%$ in the ruxolitinib group); additional reported terms were neuropathy peripheral, neuralgia, and peripheral motor neuropathy (momelotinib group only). These AEs were grade 1 to 2, with the exception of grade 3 peripheral sensory neuropathy, reported for one patient $(0.5 \%)$ in the ruxolitinib group. No patients discontinued momelotinib or ruxolitinib because of peripheral neuropathy.

A total of 17 events considered related to first-dose effects were reported for 14 patients $(6.5 \%)$ in the momelotinib group: hypotension $(3.3 \%)$, dizziness $(1.9 \%)$, flushing $(1.4 \%)$, nausea $(0.9 \%)$, and headache $(0.5 \%)$. All events considered related to first-dose effects were grade 1 to 2, with the exception of one grade 3 event (hypotension), which was also reported as serious and resulted in a dose reduction. In the ruxolitinib group, AEs considered related to first-dose effects were reported for two patients $(0.9 \%)$ who experienced grade 1 dizziness. No event in the ruxolitinib group was reported as serious.

\section{DISCUSSION}

SIMPLIFY-1 evaluated the efficacy and safety of momelotinib compared with ruxolitinib in patients who had not received prior 


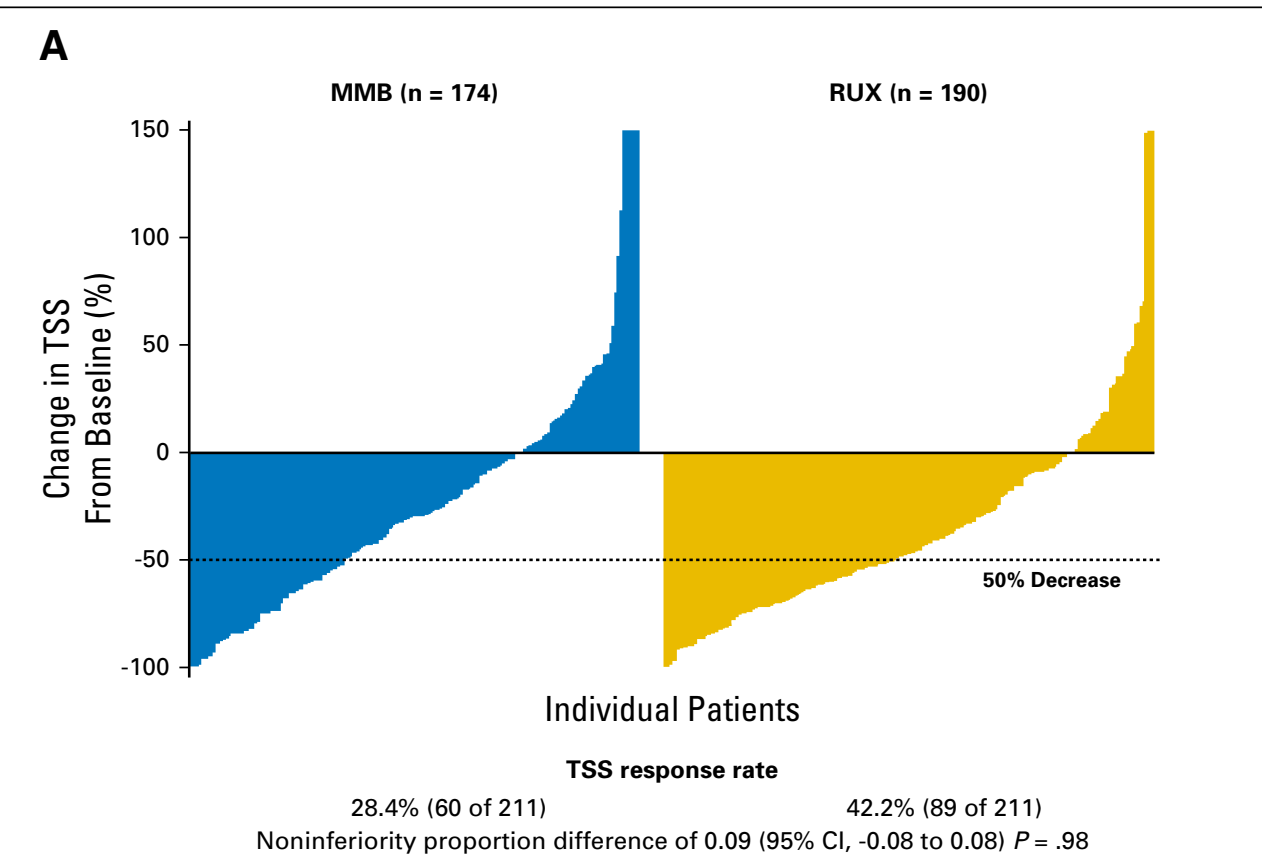

\section{B}

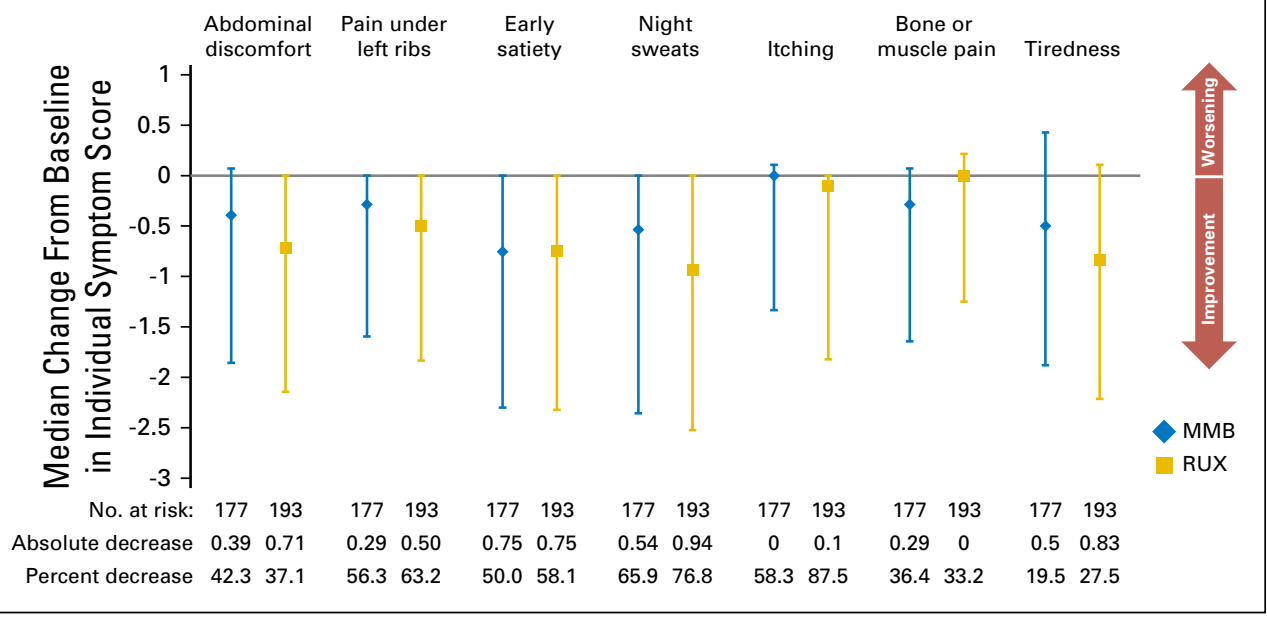

Fig 2. (A) Change in Total Symptom Score (TSS) from baseline and TSS response rate (percentage of patients with $\geq 50 \%$ reduction in TSS) at week 24. (B) Absolute and percent changes in individual symptoms of the Myeloproliferative Neoplasm Symptom Assessment Form from baseline to week 24. MMB, momelotinib; $R U X$, ruxolitinib. treatment with a JAKi. The study met the prespecified primary end point of noninferiority for spleen response but not the key secondary end point of symptomatic improvement. The proportion of patients who achieved $\mathrm{a} \geq 50 \%$ reduction in TSS with ruxolitinib in this study (42\%) was comparable to that in the registrational COMFORT-1 study (46\%), whereas momelotinib here achieved this response in only $28 \%$. Note that the COMFORT- 1 control group TSS response rate was $5 \%{ }^{7}$ The reduction in TSS by momelotinib here was also consistent with that observed in the second-line study, SIMPLIFY-2 (ClinicalTrials.gov identifier: NCT02101268). ${ }^{12}$ One possible explanation is that momelotinib and ruxolitinib differ in inhibition of cytokines mediating diseaserelated symptoms. Both drugs decreased inflammatory cytokines, but neither the baseline level of the cytokines nor the extent of decrease of the cytokines was associated with TSS response for either treatment (data not shown). Despite the anemia benefit of momelotinib, there was a greater reduction in fatigue with ruxolitinib, which suggests that fatigue is multifactorial and not only related to anemia.

Although momelotinib did not demonstrate an improvement over ruxolitinib for TSS, it did improve all three anemia end points and the composite clinical improvement (composite of SRR24, TSS response, and transfusion independence end points). Furthermore, more patients treated with momelotinib achieved two or more clinical improvements (SRR, TSS, or transfusion independence) than patients treated with ruxolitinib. Objective laboratory measures such as $\mathrm{Hb}$, serum iron, and transferrin saturation also favored momelotinib (Appendix, online only). Momelotinib is a negative regulator of hepcidin in the liver through its inhibitory effect on the ACVR1 pathway, increasing the release of iron from sequestered cellular stores and enhancing erythropoiesis in a nonclinical study. ${ }^{10}$ This may be one mechanism underlying the anemia 


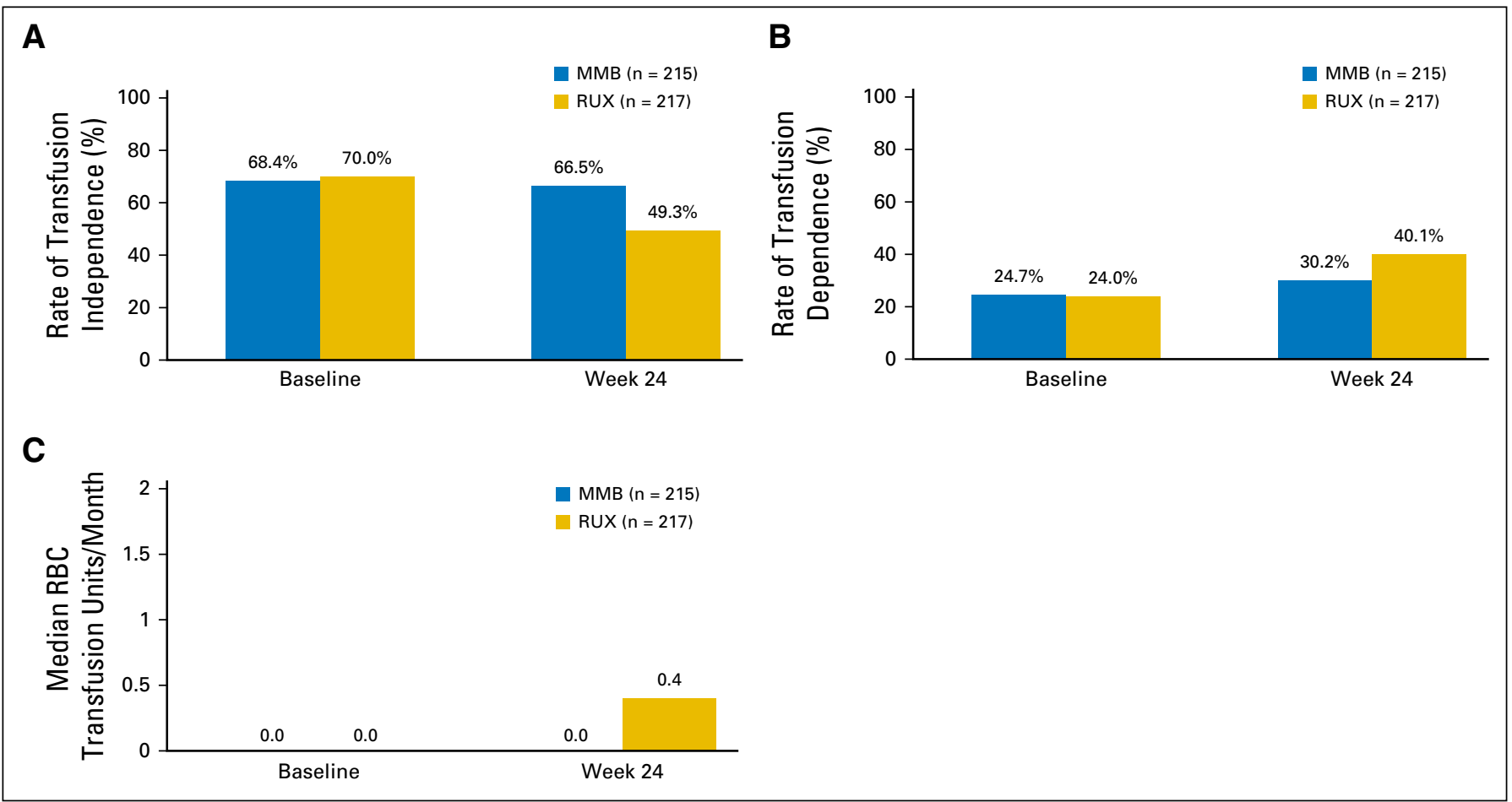

Fig 3. Comparison of momelotinib (MMB) and ruxolitinib (RUX) effects on transfusion requirements at week 24. ( $A$ ) RBC transfusion independence rate (no RBC transfusion and no hemoglobin [Hb] $<8 \mathrm{~g} / \mathrm{dL}$ in the prior 12 weeks; nominal $P<.001)$. (B) RBC transfusion dependence rate $(\geq 4$ units of $\mathrm{RBC}$ transfusion or $\mathrm{Hb}<8 \mathrm{~g} / \mathrm{dL}$ in the prior 8 weeks; nominal $P=.019$ ). (C) Rate of RBC transfusions through week 24 (nominal $P<.001$ ).

response observed in patients who received momelotinib. In contrast, ruxolitinib has no activity associated with the ACVR1 pathway.

The safety profile of momelotinib in patients with MF showed fewer events of anemia and thrombocytopenia compared with ruxolitinib and a higher incidence of nausea. Peripheral neuropathy, a predominant nonhematologic AE in earlier studies, was not substantially more common with momelotinib than with ruxolitinib, nor did it lead to discontinuation for patients in either group. The mechanism of action driving peripheral neuropathy is not known, and there do not seem to be specific risk factors for momelotinibassociated peripheral neuropathy. ${ }^{18}$

In sum, these results were mixed and indicate that although momelotinib may offer less symptom control than ruxolitinib, there is a comparable spleen response and a potential benefit in terms of anemia. Given the broad range of phenotypes in MF and

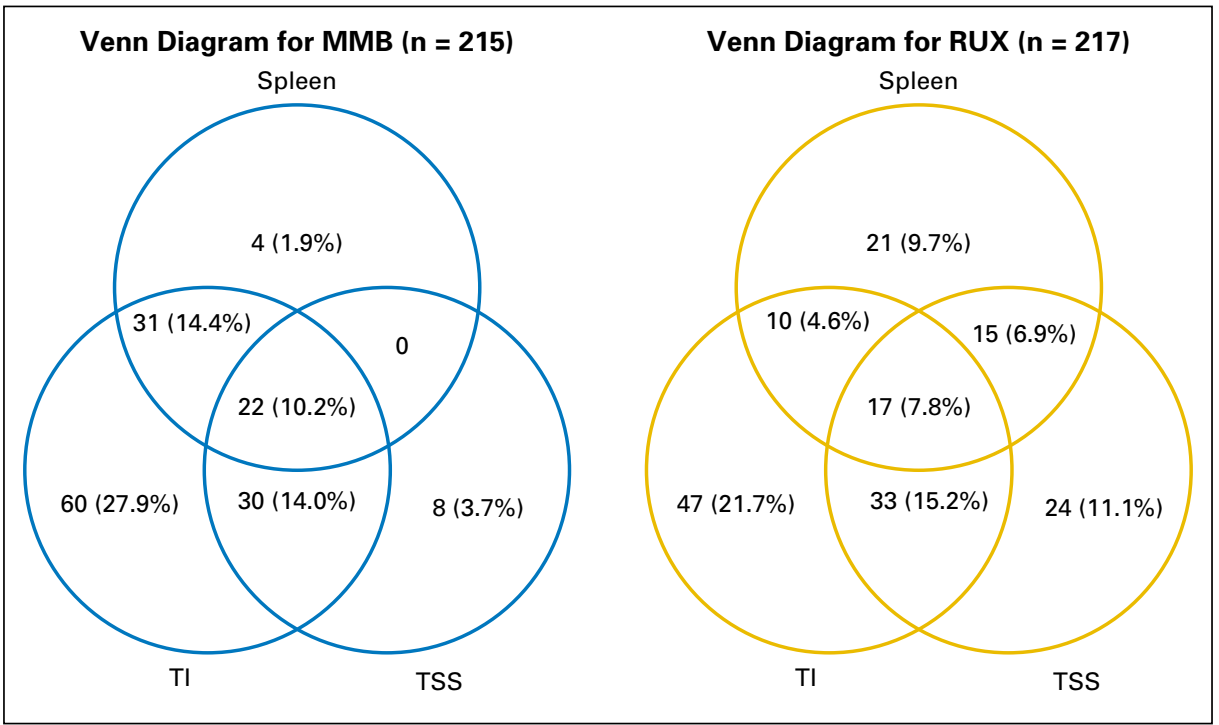

jco.org
Fig 4. Patients ( $n, \%)$ who achieved spleen response ( $\geq 35 \%$ reduction in spleen volume), Total Symptom Score (TSS) response ( $\geq 50 \%$ reduction in score), and transfusion independence ( $\mathrm{Tl}$; no $\mathrm{RBC}$ transfusion and no $\mathrm{Hb}<8 \mathrm{~g} / \mathrm{dL}$ in the prior 12 weeks) at week 24. MMB, momelotinib; RUX, ruxolitinib. 


\begin{tabular}{lcr}
\multicolumn{2}{c}{$\begin{array}{c}\text { Table 2. Treatment-Emergent Adverse Events Occurring in } \geq 10 \% \text { of Either } \\
\text { Treatment Group }\end{array}$} \\
\hline & \multicolumn{2}{c}{ Double-Blind Phase } \\
\cline { 2 - 3 } & $\begin{array}{c}\text { Momelotinib } \\
(\mathrm{n}=214)\end{array}$ & $\begin{array}{r}\text { Ruxolitinib } \\
(\mathrm{n}=216)\end{array}$ \\
\hline Treatment-Emergent Adverse Event & $40(18.7)$ & $63(29.2)$ \\
\hline Thrombocytopenia & $38(17.8)$ & $43(19.9)$ \\
Diarrhea & $37(17.3)$ & $43(19.9)$ \\
Headache & $34(15.9)$ & $25(11.6)$ \\
Dizziness & $34(15.9)$ & $8(3.7)$ \\
Nausea & $31(14.5)$ & $26(12.0)$ \\
Fatigue & $29(13.6)$ & $82(38.0)$ \\
Anemia & $22(10.3)$ & $24(11.1)$ \\
Abdominal pain &
\end{tabular}

NOTE. Data presented as No. (\%).

different treatment needs for individual patients, treatment decisions might also consider these tradeoffs, should momelotinib gain approval for this indication. Long-term efficacy and safety studies are warranted in the JAKi-naïe patient population and in patients who have received prior JAKi treatment.
AUTHORS' DISCLOSURES OF POTENTIAL CONFLICTS OF INTEREST

Disclosures provided by the authors are available with this article at jco.org.

\section{AUTHOR CONTRIBUTIONS}

Conception and design: Ruben A. Mesa, Wei Deng, Jason Gotlib Provision of study materials or patients: Ruben A. Mesa, John V. Catalano, Timothy Devos

Collection and assembly of data: Timothy Devos, Miklos Egyed, Andrzei Hellmann, Donal McLornan Kazuya Shimoda, Wei Deng, Julia D.

Maltzman, Francisco Cervantes

Data analysis and interpretation: Jean-Jacques Kiladjian, John V. Catalano, Miklos Egyed, Kazuya Shimoda, Elliott F. Winton, Wei Deng, Ronald L. Dubowy, Julia D. Maltzman, Francisco Cervantes

Manuscript writing: All authors

Final approval of manuscript: All authors

Accountable for all aspects of the work: All authors

\section{REFERENCES}

1. Pardanani $A$, Gotlib JR, Jamieson $C$, et al: Safety and efficacy of TG101348, a selective JAK2 inhibitor, in myelofibrosis. J Clin Oncol 29:789-796, 2011

2. Levine $R L$, Pardanani $A$, Tefferi $A$, et al: Role of JAK2 in the pathogenesis and therapy of myeloproliferative disorders. Nat Rev Cancer 7:673-683, 2007

3. Delhommeau F, Jeziorowska D, Marzac C, et al: Molecular aspects of myeloproliferative neoplasms. Int J Hematol 91:165-173, 2010

4. Klampfl T, Gisslinger $H$, Harutyunyan AS, et al: Somatic mutations of calreticulin in myeloproliferative neoplasms. N Engl J Med 369:2379-2390, 2013

5. Pardanani A: JAK2 inhibitor therapy in myeloproliferative disorders: Rationale, preclinical studies and ongoing clinical trials. Leukemia 22 : 23-30, 2008

6. Harrison C, Kiladjian JJ, Al-Ali HK, et al: JAK inhibition with ruxolitinib versus best available therapy for myelofibrosis. N Engl J Med 366:787-798, 2012

7. Verstovsek S, Mesa RA, Gotlib J, et al: A double-blind, placebo-controlled trial of ruxolitinib for myelofibrosis. N Engl J Med 366:799-807, 2012
8. Pardanani $A$, Lasho $T$, Smith $G$, et al: CYT387, a selective JAK1/JAK2 inhibitor: In vitro assessment of kinase selectivity and preclinical studies using cell lines and primary cells from polycythemia vera patients. Leukemia 23:1441-1445, 2009

9. Tyner JW, Bumm TG, Deininger J, et al: CYT387, a novel JAK2 inhibitor, induces hematologic responses and normalizes inflammatory cytokines in murine myeloproliferative neoplasms. Blood 115: 5232-5240, 2010

10. Asshoff $M$, Petzer V, Warr MR, et al: Momelotinib inhibits ACVR1/ALK2, decreases hepcidin production, and ameliorates anemia of chronic disease in rodents. Blood 129:1823-1830, 2017

11. Gupta $V$, Mesa RA, Deininger MWN, et al: $A$ phase $1 / 2$, open-label study evaluating twice-daily administration of momelotinib in myelofibrosis. Haematologica 102:94-102, 2017

12. Harrison $C N$, Vannucchi $A M$, Platzbecker $U$, et al: Phase 3 randomized trial of momelotinib (MMB) versus best available therapy (BAT) in patients with myelofibrosis (MF) previously treated with ruxolitinib (RUX). J Clin Oncol 35, 2017 (abstr 7001; suppl)

13. Tefferi A, Thiele J, Orazi $A$, et al: Proposals and rationale for revision of the World Health Organization diagnostic criteria for polycythemia vera, essential thrombocythemia, and primary myelofibrosis:
Recommendations from an ad hoc international expert panel. Blood 110:1092-1097, 2007

14. Barosi G, Mesa RA, Thiele J, et al: Proposed criteria for the diagnosis of post-polycythemia vera and post-essential thrombocythemia myelofibrosis: A consensus statement from the International Working Group for Myelofibrosis Research and Treatment. Leukemia 22:437-438, 2008

15. Cervantes F, Dupriez B, Pereira A, et al: New prognostic scoring system for primary myelofibrosis based on a study of the International Working Group for Myelofibrosis Research and Treatment. Blood 113:2895-2901, 2009

16. Oken MM, Creech $\mathrm{RH}$, Tormey DC, et al: Toxicity and response criteria of the Eastern Cooperative Oncology Group. Am J Clin Oncol 5: 649-655, 1982

17. Tefferi $A$, Cervantes $F$, Mesa $R$, et al: Revised response criteria for myelofibrosis: International Working Group-Myeloproliferative Neoplasms Research and Treatment (IWG-MRT) and European LeukemiaNet (ELN) consensus report. Blood 122: 1395-1398, 2013

18. Abdelrahman RA, Begna $K H, A l-K a l i ~ A$, et al: Momelotinib treatment-emergent neuropathy: Prevalence, risk factors and outcome in 100 patients with myelofibrosis. Br J Haematol 169:77-80, 2015

\section{Affiliations}

Ruben A. Mesa, Mayo Clinic Cancer Center, Scottsdale, AZ; Jean-Jacques Kiladjian, Saint-Louis Hospital (Assistance Publique-Hôpitaux de Paris) and Paris Diderot University, Paris, France; John V. Catalano, Frankston Hospital and Monash University, Melbourne, Victoria, Australia; Timothy Devos, University Hospital Leuven and Katholieke Universiteit Leuven, Leuven, Belgium; Miklos Egyed, Kaposi Mor Teaching Hospital, Kaposvar, Hungary; Andrzei Hellmann, Medical University of Gdańsk, Gdańsk, Poland; Donal McLornan, Guy's and St Thomas' National Health Service Foundation Trust, London, United Kingdom; Kazuya Shimoda, University of Miyazaki, Miyazaki, Japan; Elliott F. Winton, Emory University School of Medicine, Atlanta, GA; Wei Deng, Ronald L. Dubowy, and Julia D. Maltzman, Gilead Sciences, Foster City; Jason Gotlib, Stanford Cancer Institute, Stanford, CA; and Francisco Cervantes, Hospital Clinic, Institut D’Investigacions Biomèdiques August Pi I Sunyer, University of Barcelona, Barcelona, Spain.

Supported by Gilead Sciences, Foster City, CA. 


\section{AUTHOR'S DISCLOSURES OF POTENTIAL CONFLICTS OF INTEREST}

SIMPLIFY-1: A Phase III Randomized Trial of Momelotinib Versus Ruxolitinib in Janus Kinase Inhibitor-Naïve Patients With Myelofibrosis

The following represents disclosure information provided by authors of this manuscript. All relationships are considered compensated. Relationships are self-held unless noted. I = Immediate Family Member, Inst = My Institution. Relationships may not relate to the subject matter of this manuscript. For more information about ASCO's conflict of interest policy, please refer to www.asco.org/rwc or ascopubs.org/jco/site/ifc.

Ruben A. Mesa

Honoraria: Shire, Novartis, AOP Orphan Pharmaceuticals Consulting or Advisory Role: Galena Biopharma, Baxalta, Incyte, Novartis

Research Funding: Genentech (Inst), Incyte (Inst), CTI Biopharma (Inst), Promedior (Inst), NS Pharma (Inst), Gilead Sciences (Inst), Pfizer (Inst), PharmaEssentia (Inst), Celgene (Inst)

Travel, Accommodations, Expenses: Incyte, Novartis, AOP Orphan Pharmaceuticals

\section{Jean-Jacques Kiladjian}

Consulting or Advisory Role: AOP Orphan Pharmaceuticals, Novartis, Shire

Research Funding: AOP Orphan Pharmaceuticals (Inst), Novartis (Inst) Travel, Accommodations, Expenses: Novartis

\section{John V. Catalano}

Consulting or Advisory Role: Celgene, Gilead Sciences, Roche Travel, Accommodations, Expenses: Bristol-Myers Squibb, Celgene, Amgen

Timothy Devos

Consulting or Advisory Role: Gilead Sciences, Novartis Pharma K.K., Janssen

Miklos Egyed

No relationship to disclose

Andrzei Hellmann

Travel, Accommodations, Expenses: Bristol-Myers Squibb, ARIAD

Pharmaceuticals, Novartis Pharma K.K.

Donal McLornan

Honoraria: Jazz Pharmaceuticals

Speakers' Bureau: Novartis Pharma K.K.

\section{Kazuya Shimoda}

Honoraria: Novartis, Shire

Consulting or Advisory Role: Novartis

Research Funding: Bristol-Myers Squibb Japan, Novartis, Kirin

Pharmaceuticals, Takeda Pharmaceuticals

Elliott F. Winton

Consulting or Advisory Role: Incyte, Gilead Sciences

Research Funding: Incyte (Inst), Gilead Sciences (Inst)

Wei Deng

Employment: Gilead Sciences

Stock or Other Ownership: Gilead Sciences

Ronald L. Dubowy

Employment: Gilead Sciences

Stock or Other Ownership: Gilead Sciences

Julia D. Maltzman

Employment: Gilead Sciences

Stock or Other Ownership: Gilead Sciences

Francisco Cervantes

Consulting or Advisory Role: Gilead Sciences, ARIAD Pharmaceuticals, Pfizer, Novartis Pharma K.K.

Speakers' Bureau: Gilead Sciences, ARIAD Pharmaceuticals, Pfizer, Novartis Pharma K.K.

Jason Gotlib

Research Funding: Gilead Sciences 


\section{Acknowledgment}

Professional medical writing assistance was provided by Beth Sesler, CMPP, at Impact Communications (New York, NY).

\section{Appendix}

\section{Methods}

The Myeloproliferative Neoplasm Symptom Assessment Form (MPN-SAF) Total Symptom Score (TSS) is a validated instrument developed to assess symptom severity in patients with myeloproliferative neoplasms (Emanuel RM, et al: J Clin Oncol 30:4098-4103, 2012). The MPN-SAF TSS was originally derived from the MPN-SAF that evaluates symptom experience and quality of life among patients with MPNs as the total score of 10 symptom items from the larger measure (Scherber R, et al: Blood 118:401-408, 2011). The MPN-SAF is a modified myelofibrosis symptom assessment form that captures the symptom and effect experience of the entire spectrum of patients with MPNs, including patients with polycythemia vera and essential thrombocythemia. The 10-item MPN-SAF TSS was revised to result in the modified MPN-SAF TSS used in this study. The modified MPN-SAF TSS v2.0 used in this study contained eight questions, only seven of which were used to generate the score. The included questions were related to tiredness, early satiety, abdominal discomfort, night sweats, itching, bone pain, and pain under the ribs on the left side.

\section{Safety}

Objective laboratory measures such as hemoglobin $(\mathrm{Hb})$, serum iron, and transferrin saturation all favored momelotinib over ruxolitinib. Mean serum Hb levels were consistently higher in the momelotinib group than in the ruxolitinib group for the duration of double-blind treatment. The mean (SD) maximum percent increase in $\mathrm{Hb}$ from baseline during treatment was $15.0 \%$ (14.86\%) for the momelotinib group and 7.5\% (13.82\%) for the ruxolitinib group. At week 24 (end of treatment), the mean (SD) percent change from baseline in $\mathrm{Hb}$ was 6.9\% (16.00\%) for the momelotinib group and $-6.5 \%$ (14.74\%) for the ruxolitinib group.

Serum iron and transferrin saturation at week 24 are shown in Appendix Table A1 (online only). Compared with patients treated with momelotinib, those treated with ruxolitinib tended to have higher serum iron and transferrin saturation at week 24 . There were $9 \%$ and $23 \%$ of patients with transferrin saturation $>50 \%$ at week 24 in the momelotinib and ruxolitinib groups, respectively. 


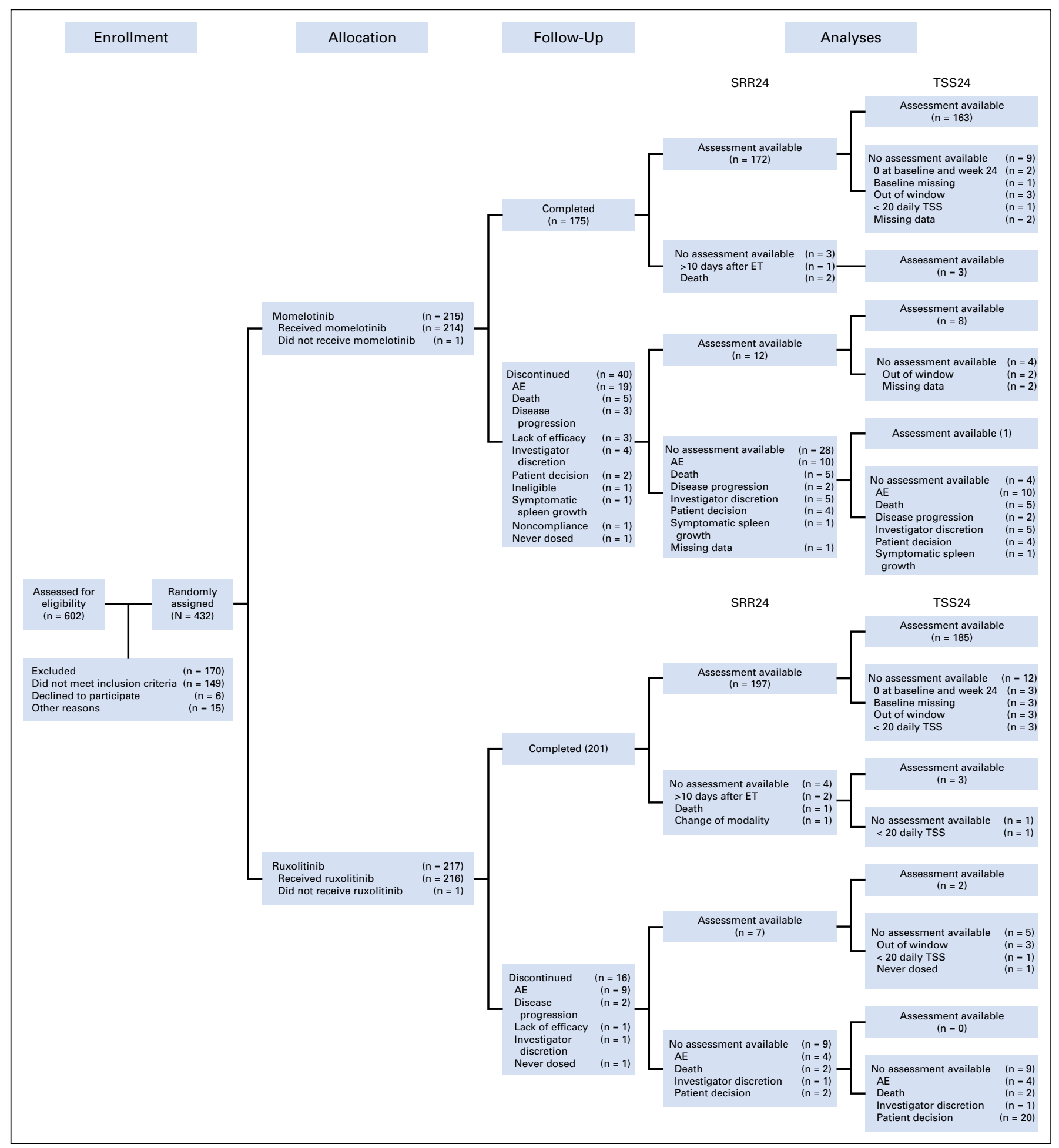

Fig A1. Patient disposition and availability for assessments. AE, adverse effect; ET, essential thrombocythemia; SSR24, spleen response rate at 24 weeks; TSS24, Total Symptom Score at 24 weeks. 